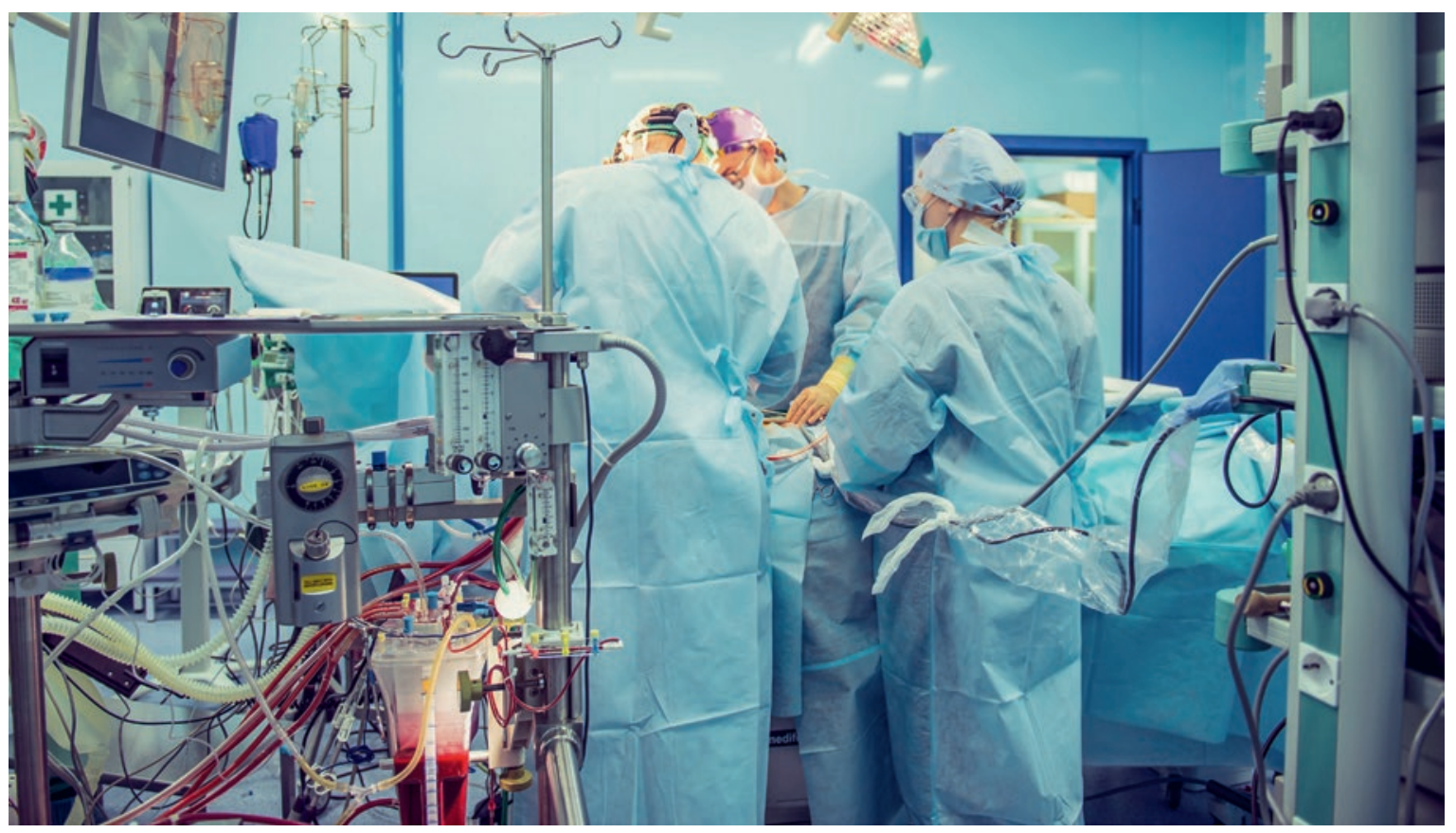

Organ- und allogene Stammzellentransplantationen werden seit 2010 der hochspezialisierten Medizin zugeordnet.

\title{
Wo steht die hochspezialisierte Medizin in der Schweiz?
}

\section{Martin F. Fey}

Prof. em. Dr. med., Präsident Fachorgan Hochspezialisierte Medizin (HSM) der Konferenz der nationalen Gesundheitsdirektorinnen und -direktoren (GDK)

Die Interessenvereinbarung zur hochspezialisierten Medizin von 2008 hält fest, dass zur Qualitätsverbesserung komplexe Eingriffe bei seltenen Erkrankungen nur wenigen Kompetenzzentren zugeteilt werden. Etabliert sind beispielsweise die jeweiligen Zentren in der Transplantationsmedizin oder der Behandlung schwerer Verbrennungen. Die hochspezialisierte Medizin ist auf Kurs und erzielt seit zehn Jahren gute Fortschritte.

Nach einer dornenreichen Vorgeschichte signierten 2008 alle Schweizer Kantone die Interessenvereinbarung zur hochspezialisierten Medizin (IVHSM), um zur Qualitätssicherung aufwendige Therapien seltener Krankheiten auf wenige grosse Spitalzentren zu be-

Zur Qualitätssicherung werden aufwendige Therapien seltener Krankheiten auf wenige grosse Spitalzentren beschränkt.

schränken. Gemäss dem Bundesgesetz über die Krankenversicherung KVG (Art. 39) kann die hochspezialisierte Medizin (HSM) nur stationäre Leistungen regeln. Die Planung hat ferner den Bedarf und eine angemes- sene geographische Verteilung der HSM-Zentren im Land zu berücksichtigen.

HSM-Beschlüsse werden vom HSM-Beschlussorgan (BO) gefasst; es setzt sich zusammen aus zehn kantonalen Gesundheitsdirektorinnen und -direktoren, u.a. aus den fünf Kantonen mit alteingesessenen Universitäten. Das Fachorgan (FO) vereinigt 15 Spezialistinnen und Spezialisten aus diversen Gebieten der Medizin. Es ist zuständig für die evidenzbasierte Aufarbeitung der HSM-Themen, u.a. der Bereiche, in denen keine spontane Selbstregulation erfolgt, aber Handlungsbedarf besteht. Unterstützt wird es durch Begleitgruppen, d.h. Expertengruppen, die durch die jeweiligen Fachgesellschaften nominiert werden. 


\section{Zuordnungs- und Zuteilungsverfahren}

Das Bundesverwaltungsgericht (BVG) schreibt ein zweistufiges HSM-Verfahren vor. Als Erstes erfolgt eine Zuordnung ("rattachement») zur HSM. Zielthemen sind gemäss der IVHSM 2008 "medizinische Bereiche und Leistungen, die durch ihre Seltenheit, ihr hohes Innovationspotenzial, durch einen hohen personellen oder technischen Aufwand und durch komplexe Behandlungsverfahren gekennzeichnet sind».

Dass eine fehlende Zuteilung zur hochspezialisierten Medizin die Attraktivität einer Klinik schwäche, konnte widerlegt werden.

Im zweiten Schritt, der Zuteilung («attribution»), wird der Leistungsauftrag an ein Spital vergeben; HSMzugeordnete Eingriffe müssen zulasten der obligatorischen Grundversicherung ausgeführt werden. Die Zuteilungskriterien werden vom FO auf wissenschaftlicher Basis festgelegt. Sie umfassen Prozess- und Strukturqualität, ferner Weiterbildung und klinische For- schung. Teilweise wird kritisiert, Letztere dürften nicht als HSM-Selektionskriterien verwendet werden, da Forschung und Weiterbildung nicht durch die Krankenversicherungen finanziert werden. Das BVG beschäftigt sich momentan mit dieser Frage. Dass eine fehlende HSM-Zuteilung die Attraktivität einer Klinik für Assistenzärztinnen und -ärzte schwäche, konnte in Zusammenarbeit mit dem Schweizerischen Institut für ärztliche Weiter- und Fortbildung (SIWF) widerlegt werden. Weiter wird geprüft, ob ein Spital, das sich um eine HSM-Zuteilung bewirbt, «wirtschaftlich» arbeitet. HSM arbeitet ferner mit dem Schweizerischen GesundheitsObservatorium (OBSAN) in Neuenburg zusammen, um demographische Entwicklungen in der Schweiz, also Fragen des Bedarfs, in die HSM-Planung einzubauen.

\section{Minimale Fallzahlen}

Nebst Kriterien für Prozess- und Strukturqualität verlangen Zuteilungen meist minimale Fallzahlen (MFZ). Viel Fachliteratur belegt, dass MFZ ein validierter Surrogatmarker für Qualität bei schwierigen chirurgi-

Tabelle 1: Zusammenfassung der HSM-Bereiche

(Status Dezember 2020 - vor 2014 erfolgte Zuordnung und Zuteilung gleichzeitig, später in einem zweistufigen Verfahren).

\begin{tabular}{|c|c|c|}
\hline HSM-Bereich & HSM-Zuordnung & HSM-Zuteilung \\
\hline Organ- und allogene Stammzelltransplantation bei Erwachsenen & $2010,2013,2016$ & 2010, 2013, 2018 - diverse Universitätskliniken und KSSG \\
\hline Cochlea-Implantate & 2010, 2013, 2019 & 2010, 2013, aktuell in Evaluation \\
\hline Schwere Verbrennungen bei Erwachsenen & $2010,2013,2016$ & $2010,2013,2019-$ CHUV und USZ \\
\hline $\begin{array}{l}\text { Schweres Trauma (schwere Einzel- oder Mehrfachverletzungen } \\
\text { mit mindestens } 20 \text { Punkten des Injury Severity Score (ISS) oder } \\
\text { Schädel-Hirn-Trauma mit einem Abbreviated Injury Score (AIS) } \geq 3 \text { ) }\end{array}$ & 2011,2015 & 2011, 2017 - 12 Traumazentren in allen Landesteilen \\
\hline $\begin{array}{l}\text { Neurochirurgie und Neuroradiologie (funktionelle Neurochirurgie, } \\
\text { Rückenmarktumoren, prächirurgische Epilepsie-Diagnostik und } \\
\text { Chirurgie der refraktären Epilepsie, komplexe neurovaskuläre Eingriffe) }\end{array}$ & 2011,2015 & 2011, aktuell in Arbeit - diverse Kliniken \\
\hline $\begin{array}{l}\text { Pädiatrie und Kinderchirurgie (u.a. Organtransplantationen, } \\
\text { schweres Trauma, Stoffwechselstörungen, Intensivpflege) }\end{array}$ & 2011 & 2011, aktuell in Revision \\
\hline $\begin{array}{l}\text { Pädiatrische Onkologie (u.a. Neuroblastom, Sarkome, ZNS-Tumoren } \\
\text { und Stammzelltransplantation) }\end{array}$ & $\begin{array}{l}\text { 2013; aktuell } \\
\text { in Revision }\end{array}$ & $\begin{array}{l}2013 \text { an diversen pädiatrischen Kliniken (SPOG-Zentren); } \\
\text { aktuell Erarbeitung der Zuteilungskriterien }\end{array}$ \\
\hline $\begin{array}{l}\text { Komplexe Viszeralchirurgie (Ösophagus- und Rektumchirurgie, } \\
\text { komplexe Leberresektionen, Pankreatektomie, komplexe bariatrische } \\
\text { Chirurgie) }\end{array}$ & 2013,2016 & $\begin{array}{l}\text { 2013: nur Rektumchirurgie und komplexe bariatrische } \\
\text { Chirurgie; 2019: Ösophagus-, Leber- und Pankreas- } \\
\text { chirurgie - diverse Unispitäler, grosse Kantons- und } \\
\text { Privatspitäler. Rektum und Bariatrie: aktuell zweite } \\
\text { Zuteilungsrunde mit verschärften Kriterien }\end{array}$ \\
\hline Komplexe Behandlung von Hirnschlägen (Strokes) & 2011, 2015 & $\begin{array}{l}2011 \text { und erneut } 2018 \\
\text { an } 10 \text { Stroke centres in allen Landesteilen }\end{array}$ \\
\hline $\begin{array}{l}\text { Urologie (Zystektomie, retroperitoneale Lymphadenektomie } \\
\text { bei Hodenkarzinom) }\end{array}$ & 2020 & Evaluation der Bewerbungen in Arbeit \\
\hline Herzunterstützungssysteme (ventricular assist devices) & 2020 & Evaluation der Bewerbungen in Arbeit \\
\hline Pädiatrische Herzchirurgie & $\begin{array}{l}\text { In Bearbeitung } \\
2020 / 21\end{array}$ & Erst anzugehen nach rechtlich gültiger Zuordnung \\
\hline $\begin{array}{l}\text { Komplexe Onko-Gynäkologie } \\
\text { (Ovarkarzinom, Zervixkarzinome, Trophoblasttumoren, } \\
\text { Vulva- und Vaginakarzinone) }\end{array}$ & $\begin{array}{l}\text { In Bearbeitung } \\
2020 / 21 \\
\text { (Vernehmlassung } \\
\text { abgeschlossen) }\end{array}$ & Erst anzugehen nach rechtlich gültiger Zuordnung \\
\hline Gefässchirurgie & $\begin{array}{l}\text { Vorbereitung einer } \\
\text { möglichen Zuord- } \\
\text { nung } 2020 / 21\end{array}$ & Erst anzugehen nach rechtlich gültiger Zuordnung \\
\hline
\end{tabular}


schen Eingriffen sind. Ihr Einsatz zur HSM-Spitalselektion ist laut BVG legitim und nötig. Die Evidenz, wonach die Behandlung von vielen statt nur wenigen Fällen pro Institution die Behandlungsqualität verbessert, ist naheliegend, und sie liegt für viele HSM-Gebiete und für ganz verschiedene Gesundheitssysteme vor, so auch für den "Sonderfall Schweiz» $[1,2]$. MFZ würden einen falschen Anreiz setzen, Patienten zu operieren, bei denen die Indikation fraglich oder gar nicht gegeben sei, nur damit die Klinik die kritische Masse an jährlichen MFZ erreiche. Dieses Argument ist

Fraglich ist, ob komplexe Eingriffe wirklich gut durchgeführt werden, wenn sie an einem Spital nur sehr selten vorgenommen werden.

bei Spitälern hinfällig, die ohnehin hohe HSM-Fallzahlen haben. Wer pro Jahr >50 Pankreatektomien durchführt, kann die geltenden MFZ von 20 Fällen auch bei strenger Indikationsstellung locker erreichen.

In einem Spital mit einer HSM-Zuteilung muss für jeden Patienten und jede Patientin der Minimaldatensatz in ein Register eingespeist werden. Bei Zystektomie wegen Blasenkarzinom werden beispielsweise postoperative Komplikationsraten, Gesamt- und rezidivfreies Überleben erfasst. Register werden mitunter als mühselige Pflichtübung wahrgenommen, und dass die HSM-Organe die Kosten nicht übernehmen, bereitet den Spitälern keine Freude. Für die Finanzierung dieser Pflichtregister, die aktuell lediglich DRG-Beiträge erhalten, müsste ein anderer Modus erwogen werden. Validierte Registerdaten, sofern vorhanden, werden für die Qualitätsüberprüfung und als Kriterien für spätere Zuteilungsrunden verwendet.

\section{Die aktuellen HSM-Bereiche}

Die Zuteilung der Organtransplantationen und der allogenen hämatopoietischen Stammzelltransplantation an auserwählte akademische Zentrumsspitäler erfolgte erstmals ab 2010, und sie wurde bisher mehrmals erneuert. Mittlerweile liegen Langzeitqualitätsdaten aus den Registern vor, die belegen, dass die Ergebnisse ausgezeichnet sind und sich im internationalen Quervergleich sehen lassen dürfen. Die validierten Registerdaten erlauben nun, Zuteilungen mit "outcome»-Daten vorzunehmen anstelle der leidigen MFZ - dies wäre denn auch das erklärte Ziel in anderen Bereichen, die noch nicht so weit sind.

Notfälle stellen besondere logistische Anforderungen an die HSM-Steuerung, da eine geographische Einschränkung des Spitalangebots die zeitgerechte Versorgung der Bevölkerung gefährden könnte. Anderer- seits ist es fraglich, ob komplexe Notfalleingriffe wirklich gut durchgeführt werden, wenn sie an einem Spital lediglich ein paarmal pro Jahr vorgenommen werden. Die HSM trägt dem Rechnung. So sind die zehn HSM-Zentren für die Therapie komplexer Hirnschläge auf alle Landesgegenden verteilt

Diverse Gebiete aus der Neurochirurgie, Neurologie und Neuroradiologie (funktionelle Neurochirurgie, komplexe neurovaskuläre Eingriffe u.a.m.) wurden erstmals im Jahre 2011 der HSM zugeordnet und gleichzeitig bestimmten Spitälern zugeteilt. Beschwerden gegen diese Beschlüsse führten zur Auflage des BVG, HSM-Verfahren zweistufig durchzuführen. Eine Folge war, dass die Zuordnung der Neurochirurgie zur HSM zwar 2015 bestätigt, die Zuteilung aber bis heute auf Eis gelegt wurde. Aktuell werden nun die Bewerbungen für neue Leistungsaufträge gesichtet.

Die HSM-Viszeralchirurgie ging ein Gebiet an, dass traditionell keine exklusive Leistung der Universitätsspitäler gewesen war. Für eine Darstellung dieser Kontroverse sei auf den Artikel verwiesen, der 2018 in diesen Spalten erschien [3]. Aktuell sind die Bereiche Ösophagus-, Pankreaschirurgie und komplexe Lebereingriffe rechtsgültig zugeteilt. Noch sind seit Frühjahr 2019 einzelne Klagen gegen diese Entscheide vor dem BVG hängig. Die Vorschläge des FO für die Zuteilung der Rektum- und der komplexen bariatrischen Chirurgie wies das BO kürzlich zurück, mit dem Auftrag, die Zuteilungskriterien zu verschärfen - zweifelsohne eine adäquate Stossrichtung.

Der Fortschritt wurde sowohl in der Neurochirurgie als auch in der Viszeralchirurgie durch Beschwerden erheblich verzögert - einerseits ein unstrittiges Recht jedes Spitals, andererseits ein bedauerlicher Mangel an Einsicht in die Notwendigkeit, via HSM-Zentralisierung die Qualität dieser Bereiche zu verbessern.

Die Langzeitqualitätsdaten belegen, dass die Ergebnisse ausgezeichnet sind und sich im internationalen Vergleich sehen lassen dürfen.

In der Urologie wurden kürzlich die Zystektomie und die retroperitoneale Lymphadenektomie wegen Hodenkarzinom-Metastasen der HSM zugeordnet. Die Begleitgruppe sowie das FO beschäftigen sich derzeit mit einem Zuteilungsvorschlag an das BO, der nach vorgegebenen Kriterien bedarfsgerecht die "winner» unter den Bewerberspitälern ausliest.

In der gynäkologischen Onkologie werden derzeit die Voten aus der Vernehmlassung eines Zuordnungsvorschlags gesichtet, der Eingriffe bei Zervix-, Vulvaund Vaginakarzinomen, bei Ovarialkarzinomen und schliesslich die stationäre Therapie der sehr seltenen 
Trophoblasttumoren vorsieht. Kritiker monieren, dass bei einer restriktiven Zuteilung dieser Eingriffe an nur wenige Spitäler die Attraktivität derjenigen abnähme, die nicht zum HSM-Handkuss kämen. Die in der Zuordnung vorgeschlagenen Eingriffe machen aber, ähnlich wie in der Viszeralchirurgie, nur wenige Prozente aller Eingriffe aus, die eine gynäkologische Klinik (ohne Einrechnung der Geburtshilfe) gemeinhin durchführt. Es fällt schwer nachzuvollziehen, weshalb eine restriktive Zuteilung dieser Eingriffe «fatale Folgen nicht nur für die unmittelbare Versorgungsstruktur, sondern auch für die Besetzung von Kaderpositionen» haben soll.

\section{Gute bis sehr gute Ergebnisse}

Die Regulation der HSM hat in den letzten Jahren an Fahrt gewonnen. Neue Gebiete wurden zugeordnet, z.B. die Urologie; alte Themen aufgefrischt, so die komplexe Neurochirurgie und die Therapie von schweren zerebrovaskulären Insulten, ferner die Pädiatrie. Dauerbrenner im guten Sinne des Wortes sind die Organtransplantationen, die Therapie schwerer Verbrennungen und die Cochlea-Implantate, ferner die HSM-Traumazentren. Die Pflichtregister mit ihren klinischen Minimaldatensätzen erlauben die Überprüfung der HSM-Leistungen in den Spitälern mit Leistungsauftrag. Im Grossen und Ganzen sind die Ergebnisse gut bis teilweise sehr gut.

Kritische Stimmen werfen der HSM unangemessene staatliche Eingriffe in die Spitalplanung vor. Ferner wird moniert, dass Kliniken angeblich um ihr Überleben bangen müssten, wenn sie keine HSM-Zuteilung erhalten. Die Behauptung, in der Schweiz sei die Behandlungsqualität auch bei kleinen Fallzahlen sehr gut, ist nicht genügend belegt [1, 2]. 2019 wurden bei- spielsweise etwa 600 Zystektomien in über 50 Schweizer Kliniken vorgenommen, in 19 Spitälern weniger als 5× pro Jahr. Es ist nicht glaubhaft, dass die Qualität der Arbeit unerfahrener Teams gleich gut sei wie die der Teams in grossen Spitalzentren, in denen diese HSMEingriffe Alltag sind. Schliesslich werden die lähmenden bürokratischen Prozesse kritisiert. Die HSM«Bürokratie» ist allerdings nicht zuletzt dem Umstand geschuldet, dass HSM-Regularien den juristisch-puristischen Vorgaben des BVG genügen müssen, um im Beschwerdefall bestehen zu können.

Die Zentralisierung der HSM in der Schweiz ist weiterhin nötig und sinnvoll; die Vernehmlassungen zu den HSM-Zuordnungen und -Zuteilungen sorgen dafür, dass die Organe der HSM nicht einfach Leistungsaufträge oktroyieren, sondern mit Transparenz HSMSpitalzentren selektionieren, um die Qualität der HSM in der Schweiz zu verbessern und auf dem erreichten hohen Niveau zu halten.

Ich danke den Herren RR Rolf Widmer (Glarus) und RR Mauro Poggia (Genf), dem Generalsekretär der GDK Michael Jordi und dem HSMProjektteam (Katharina Schönbucher, Noëlla Gérard, Luzia Guyer, Matthias Fügi) für die scharfsinnig kritische Durchsicht des Manuskripts.

\section{Bildnachweis}

(c) Oksana Krasiuk | Dreamstime.com, Symbolbild

\section{Literatur}

1 Renzulli P, Lowy A, Maibach R, Egeli RA, Metzger U, Laffer UT. The influence of the surgeon's and the hospital's caseload on survival and local recurrence after colorectal cancer surgery. Surgery. 2006;139:296-304.

2 Güller U, Warschkow R, Ackermann CJ, Schmied B, Cerny T, Ess S Lower hospital volume is associated with higher mortality after oesophageal, gastric, pancreatic and rectal cancer resection. Swiss Med Wkly. 2017;147:w14473.

3 Fey MF. Hochspezialisierte Viszeralchirurgie: Zentralisiert oder Carte blanche? Schweiz Ärzteztg. 2018;99:24-7.
Prof. em. Martin Fey Universitätsklinik fü Medizinische Onkologie Inselspital und Universität CH-3010 Bern Martin.fey[at]insel.ch Tel. 0316322243 (nur dienstags)

\section{Das Wichtigste in Kürze}

- Um die Qualität von Leistungen der hochspezialisierten Medizin (HSM) sicherzustellen, werden komplexe Eingriffe auf wenige Kompetenzzentren verteilt.

- Die Kritik, dass Kliniken ohne HSM-Zuteilung benachteiligt würden, hält einer genaueren Überprüfung nicht stand. Auch die Behauptung, in der Schweiz sei die Behandlungsqualität auch bei kleinen Fallzahlen sehr gut, ist nicht genügend belegt.

\section{L'essentiel en bref}

- Pour garantir la qualité des services de médecine hautement spécialisée (MHS), les interventions complexes sont réparties entre un petit nombre de centres de compétence.

- La critique selon laquelle les cliniques sans attribution relative à la MHS seraient désavantagées est sans force probante. L'affirmation selon laquelle la qualité des soins en Suisse est très bonne même avec un petit nombre de cas n'est pas non plus suffisamment étayée. 